Rev. Int. Contam. Ambie. 33 (3) 377-391, 2017

DOI: 10.20937/RICA.2017.33.03.02

\title{
PERSPECTIVAS AMBIENTALES SOBRE LA CONTAMINACIÓN Y LA RECUPERACIÓN DEL RÍO MAGDALENA EN LA CIUDAD DE MÉXICO
}

\author{
Manuel PERLÓ COHEN y Itzkuauhtli ZAMORA SAENZ*
}

Instituto de Investigaciones Sociales, Universidad Nacional Autónoma de México. Circuito Mario de la Cueva. Ciudad de la Investigación en Humanidades, Ciudad Universitaria, Delegación Coyoacán, Ciudad de México, México, C. P. 04510

*Autor para correspondencia: itzaben@gmail.com

(Recibido mayo 2016; aceptado octubre 2016)

Palabras clave: ríos urbanos, perspectivas ambientales, rehabilitación de río, participación ciudadana

\section{RESUMEN}

Esta investigación presenta los resultados de una encuesta $(n=270)$ en la microcuenca del río Magdalena ubicada en el surponiente de la Ciudad de México, en la cual se muestran las perspectivas ambientales que tiene la población a partir de sus percepciones, valores y actitudes en torno a la contaminación y recuperación del río. El estudio establece que la mayoría de los encuestados se sienten afectados por la contaminación del río, ya que la consideran como una pérdida del patrimonio natural de la ciudad. No obstante, menos de la mitad asumieron parte de la responsabilidad por la degradación del río y no han realizado algún tipo de acción para limpiarlo. También destaca que la población considera que las acciones más importantes para recuperarlo son el mejoramiento de la educación ambiental y la ampliación de mecanismos de participación ciudadana. No obstante, son profundamente escépticos de la eficacia y la fiabilidad de los espacios participativos abiertos por el gobierno, puesto que los consideran manipulados para legitimar decisiones previamente tomadas por la misma autoridad. La variable sociodemográfica que tuvo mayor peso en diferentes correlaciones estadísticas fue la ubicación geográfica en la microcuenca, de manera que en la parte baja habitan quienes se sienten más perjudicados por la contaminación del río. Los jóvenes fueron quienes más valoraron la educación ambiental como el instrumento de cambio para recuperarlo como un espacio público y ambiental de la ciudad.

Keywords: urban rivers, environmental perspectives, river rehabilitation, citizen participation

\begin{abstract}
This research shows the main results of a survey $(n=270)$ in the Magdalena river micro basin located in the southwest of Mexico City. The survey identified the environmental perspectives that the population has through its perceptions, values and attitudes about pollution and river rehabilitation. The article establishes that the majority of the population feels affected for the river pollution and this fact is framed as a natural heritage loss of the city. However, less than the half survey participants share any kind of responsibility for the river degradation and have never done anything to contribute to its cleaning. In other tendencies, the population considers that the most important actions to rehabilitate the river are environmental education improvement and
\end{abstract}


the growth of citizen participation mechanisms. Nevertheless, they are pretty skeptical about the effectiveness and truthfulness of the participative spaces created by the government, because citizens consider that these spaces are manipulated in order to legitimize decisions previously taken by the same authority. The socio-demographic variable that had more weight in several statistical correlations was the geographical location in the micro basin, indeed in the lower watershed are the citizens that feel more wronged by the pollution of the river. Young people place more value on environmental education as the instrument of change to recover the river as a public and environmental space of the city.

\section{INTRODUCCIÓN}

En los últimos años han aumentado significativamente los proyectos gubernamentales dirigidos a recuperar la calidad ambiental de los ríos urbanos en varias ciudades del mundo. Este auge obedece a que dichas iniciativas permiten crear espacios públicos al interior de la traza urbana que provean de servicios ecosistémicos a la urbe, recuperar la calidad de cuerpos de agua para usos recreativos o de reúso, prevenir inundaciones mediante la restauración ecológica del cauce y revalorar económicamente ciertas zonas de la ciudad mediante la recuperación del paisaje fluvial y urbano (Riley 1998, Lundy y Wade 2001, Postel y Richter 2003, Wohl et al. 2005, Dufour y Piégay 2009, Perló 2010, Feld et al. 2011). Los ríos recuperados son altamente apreciados por la población local, convirtiéndolos en espacios de convivencia y recreación de uso frecuente (Tunstall et al. 2000, Asakawa et al. 2004). No obstante, aún son escasos los proyectos que involucran a las comunidades próximas al río en el diseño, la implementación y monitoreo de los proyectos, a pesar de que la participación ciudadana ha sido identificada como un aspecto central para la continuidad de estas políticas públicas en el mediano y largo plazos (Tapsell 1995, Postel y Richter 2003, Petts 2006 y 2007). Promover la participación ciudadana en la recuperación de un río urbano no es una tarea sencilla, ya que en la cuenca coexiste una heterogeneidad de actores que se diferencian por las prácticas y los valores que tienen con respecto al río, lo cual en principio delinea una cierta dificultad para coordinar los diferentes intereses y expectativas sobre su recuperación (Jones 1999, Perló y González 2009). Por lo tanto, un paso importante para construir la factibilidad social y política de este tipo de proyectos consiste en identificar las percepciones sociales que existen sobre el río para saber si sus condiciones actuales son enmarcadas como un problema que debe ser resuelto por la comunidad, ya que un problema ambiental sólo se constituye plenamente como tal cuando una sociedad así lo significa y por lo tanto establece la necesidad e importancia de remediarlo (Freudenburg et al. 1995, Hannigan 2006). Esta premisa no niega que el mundo biofísico y sus procesos son independientes de las construcciones sociales, pero subraya que la percepción de los problemas ambientales son cultural e históricamente contingentes (Macnaghten y Urry 1995, Burningham y Cooper 1999, Sutton 2004). Por ejemplo, Perló y González (2006) señalan cómo las percepciones sociales sobre el agua han sido determinantes en su gestión en la Cuenca de México, las que han transitado de un marco de significado caracterizado por la amenaza de la "abundancia" a uno de riesgo por la "escasez".

Las investigaciones que toman en cuenta la dimensión cognitiva de las comunidades locales para la recuperación de ríos urbanos se han centrado en las percepciones sobre la contaminación y el riesgo por inundaciones (Green y Tunstall 1992, Tunstall et al. 1994, Gobster 1998, Figuereido et al. 2009, Benez et al. 2010), ya que éstas suelen ser las motivaciones sociales más importantes para apoyar e involucrarse en este tipo de proyectos (House y Fordham 1997, Pahl-Wostl 2006). En la presente investigación se utiliza el concepto de perspectivas ambientales por considerarlo un concepto más amplio que permite incluir no sólo las percepciones sociales que tienen los habitantes acerca de los problemas de contaminación que aquejan a determinado ecosistema, también se extiende sobre un campo interpretativo que recupera los valores y las actitudes de los propios actores sociales sobre el sistema socioambiental (Durand 2008, Ímaz et al. 2015). La premisa teórica de la investigación consiste en afirmar que si bien la disposición para resolver un problema ambiental comienza con percibirlo como tal, esta percepción tiene mayores probabilidades de generar un cambio en las prácticas sociales si conlleva una transformación de valores y actitudes relacionados con el ambiente y con la participación ciudadana en la búsqueda de soluciones a lo que se ha definido como un problema ambiental de carácter público (Mohai 1985, Gould 1993, Guagnano 1995). Es decir, en la reconstrucción 
de las perspectivas ambientales de una sociedad no basta con identificar las construcciones sociales que hay sobre la naturaleza o el medio biofísico, también es importante recuperar los marcos cognitivos que orientan la acción a partir de la percepción y la valoración que hace el actor de su entorno social y político.

El estudio de caso consiste en la implementación y análisis de una encuesta que identificó las perspectivas ambientales que tienen los habitantes de la microcuenca del río Magdalena de la Ciudad de México sobre el estado del río y sobre su disposición a involucrarse en su recuperación. Además de la importancia que representa el río Magdalena como el único afluente que aporta agua potable a la ciudad (Legorreta 2009), éste ha tenido una cierta relevancia en la opinión pública capitalina a partir del intento de la pasada administración (2006-2012) de recuperarlo a partir del Plan Maestro de Manejo Integral y Aprovechamiento Sustentable, en adelante Plan Maestro (Sedema y UNAM 2009). Es importante advertir que el estudio no incluyó la dimensión pragmática o material de las perspectivas ambientales normalmente recuperados en las variables de prácticas o comportamientos, de manera que se circunscribe a los factores subjetivos que tienen los habitantes del área de estudio en torno al río Magdalena.

\section{MATERIALES Y MÉTODOS}

Área de estudio. La microcuenca del río Magdalena se ubica al surponiente de la Ciudad de México. El río nace en el paraje de Cieneguillas, a una elevación de $3650 \mathrm{msnm}$, y atraviesa el Parque Nacional de los Dinamos hasta ser captado por una planta potabilizadora que distribuye $200 \mathrm{~L} / \mathrm{s}$ para contribuir en el abastecimiento de agua de la Delegación Magdalena Contreras. Si consideramos su extensión desde su nacimiento hasta la confluencia con el río Eslava, el río tiene una extensión de $21.6 \mathrm{~km}$ (Carmona et al. 2016), pero si consideramos la longitud del nacimiento hasta la confluencia del río Mixcoac, éste alcanzaría los $28.2 \mathrm{~km}$ (Sedema y UNAM 2009). El suelo de conservación de la microcuenca se caracteriza por la presencia de vegetación representativa de la Provincia Florística de las Serranías Meridionales de la Región Mesoamericana de Montaña, particularmente de comunidades vegetales de Pinus hartwegii, Abies religiosa y bosque mixto (Almeida-Leñero et al. 2007). Los graves problemas de contaminación del río comienzan después de la planta potabilizadora, ya que el crecimiento urbano ha utilizado el cauce como depósito de aguas negras y residuos sólidos, además la planicie de inundación ha sido ocupada casi en su totalidad para la construcción de vivienda. Es importante advertir que las principales obras del Plan Maestro se realizaron en esta zona en los años 2011 y 2012, de manera que se disminuyó sensiblemente la contaminación por aguas residuales debido a la construcción de un colector central. Al momento de levantar la encuesta estas obras todavía no transformaban el paisaje de la zona, aunque tampoco se expresaban en nuevas externalidades como la desecación del cauce en estiaje y nuevas zonas de inundación en temporada de lluvias.

El río desemboca en la Presa Anzaldo, infraestructura hidráulica que deriva las avenidas extraordinarias de este río junto con las de Texmaloya, Texcalatlaco y Coyotes hacia el Interceptor poniente. En estiaje el río recorre su antiguo cauce actualmente entubado y recolecta el agua residual de las colonias aledañas a la avenida Río Magdalena y Paseo del Río en Chimalistac (Fig.1). El río vuelve a recuperar el cauce abierto a la altura del puente de Panzacola en la Delegación Coyoacán, atraviesa los Viveros de Coyoacán y un conjunto habitacional hasta confluir con el río Mixcoac y formar el río Churubusco.

Muestra. El cálculo del tamaño de muestra utilizó como población total a las personas que habitan la microcuenca (107 294) de acuerdo con la delimitación del área urbana de la cuenca que utilizó el Plan Maestro y que consideró la población de las áreas geoestadísticas básicas (AGEB) que se encuentran en el área de estudio. Para el cálculo del tamaño de muestra (n), se utilizó un margen de error de $5 \%$ y un nivel de confianza de $90 \%$, al desconocer la proporción esperada se utilizó el valor de $50 \%$ :

$\mathrm{n}=\frac{Z^{2} P(1-P) N}{(N-1) e^{2}+z^{2} p(1-p)}$

En donde $\mathrm{n}=$ tamaño de la muestra, $\mathrm{Z}=$ valor que corresponde al nivel de confianza elegido $(90 \%$ $=1.645), \mathrm{p}=$ proporción esperada, $\mathrm{N}=$ tamaño de la población y e = error máximo. Sustituyendo valores tenemos que:

$$
\frac{1.645^{2} 0.5(1-0.5) 107294}{107294-1) 0.05^{2}+}=\begin{aligned}
& 269.92 \\
& \text { (redondeo, 270) } \\
& 1.645^{2} 0.5(1-0.5)
\end{aligned}
$$

En estudios similares se identificó la importancia que tiene la ubicación geográfica del actor en sus percepciones sobre el río (Norton y Hannon 1997, Ryan 1998, Brody et al. 2004, Benez et al. 2010), de 


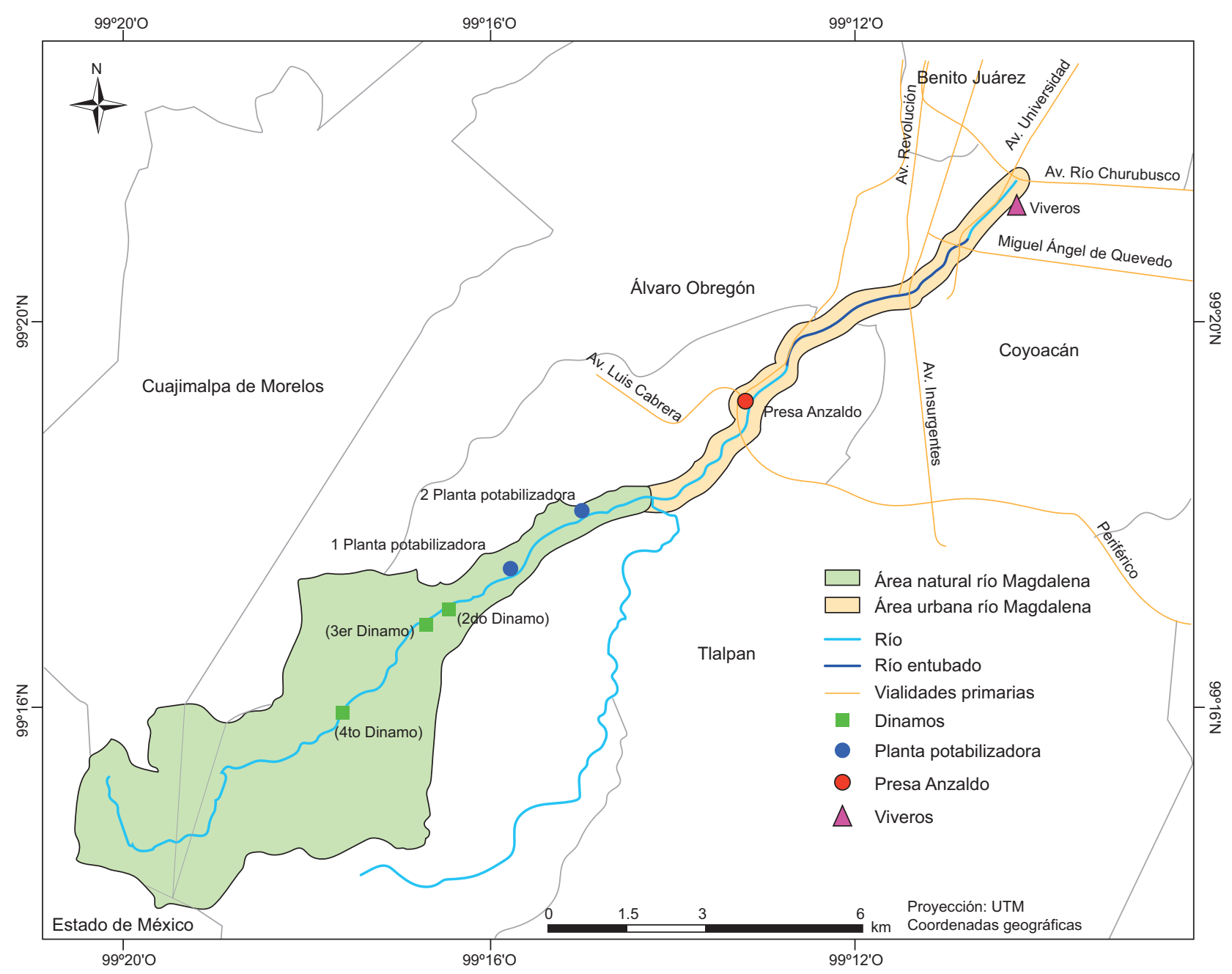

Fig. 1. Delimitación del área de estudio Fuente: Elaboración propia

manera que para el levantamiento de la encuesta la microcuenca se dividió en parte alta (de Cieneguillas hasta la Presa Anzaldo) y parte baja (después de la Presa Anzaldo hasta la avenida Río Churubusco) con el objetivo de asignar en partes iguales el número de encuesta en ambas partes y evitar una sobre representación de la parte baja que tiene una mayor consolidación urbana y por ende, mayor densidad poblacional. Posteriormente, el proceso de selección de los elementos de la muestra se realizó mediante un muestreo estratificado por asignación proporcional (Pérez 2005) de acuerdo con el tamaño de la población de cada una de las AGEB.

Instrumento. El cuestionario estuvo organizado para identificar tres dimensiones analíticas de las perspectivas socioambientales de la población encuestada: a) Percepciones. La manera en que el sujeto identifica el estado actual del río, sus problemas y el Plan Maestro que pretende recuperarlo, dicho conocimiento es resultado de su experiencia personal, de sus relaciones sociales o de diferentes fuentes de información (Valencia et al. 2010).

b) Valores. Reglas generales de mayor duración que las percepciones y las actitudes con las cuales el actor se relaciona con su entorno natural y sociopolítico, que le permiten calcular su involucramiento en la recuperación del río.

c) Actitudes. Evaluaciones positivas o negativas que realiza el actor a un proceso o entidad particular y que generan cierta disposición o inclinación (Petty et al. 1997, Eagly y Chaiken 1993), en este caso hacia los otros actores sociales con los que coincide en la cuenca y hacia el futuro del río. 
Las preguntas ordenadas de acuerdo con su dimensión se presentan en el cuadro I. En los cuadros II y III se muestran los principales resultados de la encuesta. Cuando las respuestas podían organizarse en tablas de 2 X 2 como en las preguntas B, D, E, K y $\mathrm{O}$, se realizaron pruebas de hipótesis de Chi-cuadrado para identificar posibles dependencias entre las principales respuestas obtenidas en cada pregunta y las variables sociodemográficas de ubicación en la microcuenca (parte alta y baja), edad, sexo, escolaridad y tiempo de residencia (Cuadro IV). La información estadística descriptiva e inferencial se procesó con el programa IBM SPSS Statistics, versión 20.

CUADRO I. PREGUNTAS DE LAENCUESTA POR DIMENSIÓN ANALÍTICA

\begin{tabular}{l}
\hline \multicolumn{1}{c}{ PERCEPCIONES } \\
\hline A. ¿Cuáles serían las tres palabras con las que describiría al río \\
Magdalena? \\
B. ¿A usted le afecta la contaminación del río? ¿Cómo? \\
C. ¿Quién sería el responsable de que el río se encuentre en ese \\
estado? ¿Por qué? \\
D. ¿Considera que usted también es responsable del estado en \\
el que se encuentra el río? ¿Por qué? \\
E. ¿Sabe del plan para rescatar el río? \\
F. ¿Cómo se enteró?
\end{tabular}

\section{VALORES}

G. ¿Qué medida sería la más importante para recuperar la calidad ambiental del río? ¿Por qué?

H. ¿Qué sería necesario para que usted participe en la recuperación del río?

I. ¿Cuál sería la principal ventaja de participar en el rescate?

J. ¿Cuál sería la principal desventaja de participar en el rescate?

\section{ACTITUDES}

K. ¿Hace o ha hecho algo para mejorar la calidad del río? ¿Qué?

L. ¿Quién es el actor menos confiable para recuperar el río? ¿Por qué?

M. ¿Quién es el actor más confiable para recuperar el río? ¿Por qué?

N. ¿Qué estaría dispuesto a dar o hacer si eso garantiza la limpieza del río?

O. ¿Cree que realmente el río Magdalena estará limpio algún día? ¿Por qué?

Fuente: Elaboración propia

\section{RESULTADOS}

En la muestra $52.2 \%$ de los encuestados fueron mujeres, el rango de edad que más participó fue de 50 a 69 años con $33 \%$ (media muestral, 44.38 años), la mayoría tiene estudios equivalentes al bachillerato,
CUADRO II. PERFIL SOCIODEMOGRÁFICO DEL UNIVERSO ENCUESTADO (\%)

\begin{tabular}{lccc}
\hline Educación & \multicolumn{3}{c}{ Tiempo de residencia } \\
\hline Primaria & 13.3 & 11 a 20 años & 18.1 \\
Secundaria & 17.4 & 21 a 30 años & 22.6 \\
Media superior & 33 & 31 a 40 años & 16.3 \\
Licenciatura & 33 & 41 a 50 años & 23 \\
Posgrado & 2.6 & 50 o más & 9.6 \\
N/C & 0.35 & & \\
\hline
\end{tabular}

Fuente: Elaboración propia con base en trabajo de campo. Tamaño de muestra $=270$. La ubicación en la cuenca es realizó en partes iguales, $50 \%$ en la alta y $50 \%$ en la baja. $\mathrm{N} / \mathrm{C}=$ no contestó

carrera técnica o licenciatura (66\%) y $22.6 \%$ habita la microcuenca de 21 a 30 años (media muestral, 31.27 años). Las palabras con mayor frecuencia que sintetizan las percepciones sociales que tienen los encuestados sobre el río Magdalena se refieren principalmente a sustantivos o adjetivos de carácter negativo como Sucio / Un poco sucio (108 menciones), Basura / Con basura (93), Mal olor / Oloroso / Apestoso (59) y Contaminado (55). Las percepciones positivas con más menciones fueron Bonito (18) y Natural (10), las cuales se dieron principalmente en la parte alta de la cuenca. En esta dimensión se aprecia que la mayor parte de los habitantes de la microcuenca se sienten directamente afectados por la contaminación del río Magdalena ( $87.4 \%$ ). La única relación estadísticamente significativa fue con la variable de ubicación en la cuenca, de manera que quienes habitan en la parte baja de la cuenca suelen sentirse más perjudicados por la degradación del río. La afectación se enmarca en primer lugar como una pérdida de patrimonio natural en la ciudad, particularmente del río, la flora y la fauna nativa, situación que deteriora la calidad de vida de los habitantes (32.6\%). En ese sentido, el río se considera un bien público de la ciudad, el cual se debe defender porque constituye una fuente natural que aporta agua, mejora la calidad del aire y brinda espacios verdes a los capitalinos. Otras afectaciones están más relacionadas con las externalidades negativas que tiene un río contaminado como el mal olor $(17.4 \%)$, la contaminación del agua (11.1\%), amenazas a la salud por focos de infección (10\%), el deterioro del paisaje $(7 \%)$ y el peligro de inundaciones $(4.1 \%)$. La ciudadanía señala que los principales responsables de la contaminación del río son principalmente los veci$\operatorname{nos}(25.6 \%)$, las autoridades delegacionales $(23.1 \%)$ y los asentamientos irregulares (17.3\%). Los primeros porque arrojan basura en el cauce y en sus márgenes, además no muestran interés para involucrarse en su recuperación. En segundo lugar, las autoridades serían 
CUADRO III. PRINCIPALES RESULTADOS ESTADÍSTICOS DE LA ENCUESTA (Continuación)

\begin{tabular}{|c|c|c|c|c|c|}
\hline \multicolumn{6}{|c|}{ PERCEPCIONES } \\
\hline \multicolumn{3}{|c|}{$\begin{array}{l}\text { A. ¿Cuáles serían las tres palabras con las } \\
\text { que describiría al río Magdalena?* }\end{array}$} & \multicolumn{3}{|c|}{ B. ¿A usted le afecta la contaminación del río? } \\
\hline \multicolumn{2}{|l|}{ Respuesta } & $\begin{array}{l}\text { Frecuencia } \\
\text { absoluta }\end{array}$ & Respuesta & $\begin{array}{l}\text { Frecuencia } \\
\text { absoluta }\end{array}$ & $\begin{array}{l}\text { Porcentaje } \\
\quad(\%)\end{array}$ \\
\hline \multicolumn{2}{|l|}{ Sucio / un poco sucio } & 108 & Sí, mucho & 121 & 44.8 \\
\hline \multicolumn{2}{|l|}{ Basura / con basura } & 93 & Sí, poco & 115 & 42.6 \\
\hline \multicolumn{2}{|l|}{ Mal olor / apestoso / oloroso } & 59 & No & 18 & 6.7 \\
\hline \multicolumn{2}{|l|}{ Contaminado } & 55 & No lo sé & 13 & 4.8 \\
\hline \multicolumn{2}{|l|}{ Bonito } & 18 & $\mathrm{~N} / \mathrm{C}$ & 3 & 1.1 \\
\hline \multicolumn{2}{|l|}{ Grande } & 18 & Total & 270 & 100 \\
\hline \multicolumn{2}{|l|}{ Feo } & 18 & & & \\
\hline \multicolumn{2}{|l|}{ Desagüe / aguas negras } & 17 & ¿Cómo le afecta? (B bis) & & \\
\hline \multicolumn{2}{|l|}{ Ratas } & 15 & Respuesta & $\begin{array}{l}\text { Frecuencia } \\
\text { absoluta }\end{array}$ & $\begin{array}{l}\text { Porcentaje } \\
\quad(\%)\end{array}$ \\
\hline \multicolumn{2}{|l|}{ Descuidado } & 13 & $\begin{array}{l}\text { Pérdida de patrimonio natural e } \\
\text { histórico }\end{array}$ & 88 & 32.6 \\
\hline \multicolumn{2}{|l|}{ Lodo } & 12 & Mal olor & 47 & 17.4 \\
\hline \multicolumn{2}{|l|}{ Entubado } & 12 & Contaminación de agua & 30 & 11.2 \\
\hline \multirow{5}{*}{\multicolumn{2}{|c|}{ Natural }} & 10 & Riesgo para la salud & 27 & 10 \\
\hline & & & Deterioro del paisaje & 19 & 7 \\
\hline & & & Peligro de inundaciones & 11 & 4.1 \\
\hline & & & No me afecta & 48 & 17.7 \\
\hline & & & Total & 270 & 100 \\
\hline \multicolumn{3}{|c|}{$\begin{array}{l}\text { C. ¿Quién sería el responsable de que el } \\
\text { rio se encuentre en ese estado?** }\end{array}$} & \multicolumn{3}{|c|}{$\begin{array}{l}\text { D. ¿Considera que usted también es responsable del esta- } \\
\text { do en el que se encuentra el río? }\end{array}$} \\
\hline Respuesta & $\begin{array}{l}\text { Frecuencia } \\
\text { absoluta }\end{array}$ & $\begin{array}{l}\text { Porcentaje } \\
\quad(\%)\end{array}$ & Respuesta & $\begin{array}{l}\text { Frecuencia } \\
\text { absoluta }\end{array}$ & $\begin{array}{l}\text { Porcentaje } \\
\quad(\%)\end{array}$ \\
\hline Vecinos del río & 163 & 25.6 & Sí & 126 & 46.7 \\
\hline Gobierno delegacional & 147 & 23.1 & No & 144 & 53.3 \\
\hline Asentamientos irregulares & 110 & 17.3 & Total & 270 & 100 \\
\hline Turistas Parque Dinamos & 83 & 13.0 & & & \\
\hline GDF & 78 & 12.2 & \multirow{2}{*}{\multicolumn{3}{|c|}{$\begin{array}{l}\text { Soy o no soy corresponsable del río contaminado por- } \\
\text { que... (D Bis) }\end{array}$}} \\
\hline Otros & 56 & 8.8 & & & \\
\hline \multirow[t]{2}{*}{ Total } & 637 & 100 & Respuesta & $\begin{array}{l}\text { Frecuencia } \\
\text { absoluta }\end{array}$ & $\begin{array}{l}\text { Porcentaje } \\
\quad(\%)\end{array}$ \\
\hline & & & $\begin{array}{l}\text { Sí. He tirado basura en el cauce o } \\
\text { en las cercanías }\end{array}$ & 67 & 24.8 \\
\hline \multicolumn{3}{|c|}{ E. ¿Sabe del plan para rescatar el río? } & Sí. No he hecho para limpiarlo & 41 & 15.2 \\
\hline Respuesta & $\begin{array}{l}\text { Frecuencia } \\
\text { absoluta }\end{array}$ & $\begin{array}{l}\text { Porcentaje } \\
\quad(\%)\end{array}$ & Sí. Mi drenaje va al río & 16 & 6 \\
\hline Sí & 76 & 28.1 & No. Cuido el río y no contamino & 110 & 40.7 \\
\hline No & 191 & 70.7 & No. No es mi responsabilidad & 26 & 9.6 \\
\hline $\mathrm{N} / \mathrm{C}$ & 3 & 1.2 & $\mathrm{~N} / \mathrm{C}$ & 10 & 3.7 \\
\hline Total & 270 & 100 & Total & 270 & 100 \\
\hline
\end{tabular}

Fuente: Elaboración propia con base en el trabajo de campo. N/C = no contestó

*Sólo se incluyeron las respuestas con una frecuencia mayor o igual a 10

**Se permitió al encuestado señalar más de una respuesta 
CUADRO III. PRINCIPALES RESULTADOS ESTADÍSTICOS DE LA ENCUESTA (Continuación)

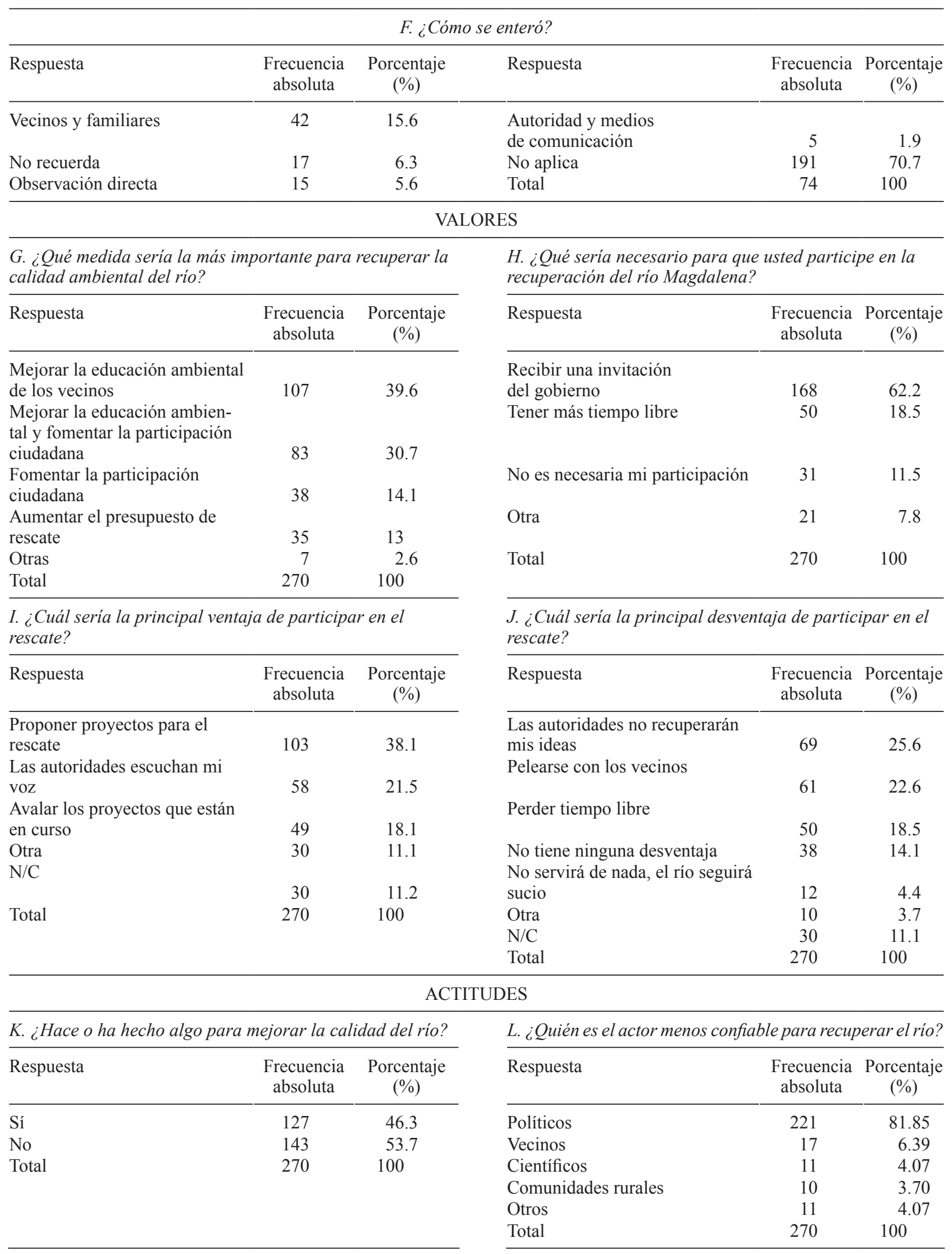

Fuente: Elaboración propia con base en el trabajo de campo. N/C = no contestó 
CUADRO III. PRINCIPALES RESULTADOS ESTADÍSTICOS DE LA ENCUESTA (Continuación)

\begin{tabular}{lcc}
$\begin{array}{l}\text { ¿Por qué los políticos le resultan los menos confiables para } \\
\text { recuperar el río? (L Bis) }\end{array}$ & $\begin{array}{c}\text { Frecuencia } \\
\text { absoluta }\end{array}$ & $\begin{array}{c}\text { Porcentaje } \\
(\%)\end{array}$ \\
\hline Respuesta & 78 & 28.9 \\
\cline { 2 - 3 } Desempeño laboral ineficiente & & \\
Prevalencia de su interés pri- & 61 & 22.6 \\
vado & 37 & 13.7 \\
Mala reputación & 32 & 11.9 \\
No les interesa el río & 13 & 4.8 \\
Otra & 49 & 18.1 \\
No aplica & 270 & 100 \\
Total & & \\
\end{tabular}

¿Por qué serían los vecinos los actores más confiables para recuperar el río? (M Bis)

\begin{tabular}{|c|c|c|c|c|c|}
\hline Respuesta & $\begin{array}{l}\text { Frecuencia } \\
\text { absoluta }\end{array}$ & $\begin{array}{l}\text { Porcentaje } \\
\quad(\%)\end{array}$ & Respuesta & $\begin{array}{l}\text { Frecuencia } \\
\text { absoluta }\end{array}$ & $\begin{array}{l}\text { Porcentaje } \\
\quad(\%)\end{array}$ \\
\hline \multicolumn{3}{|l|}{ Son los principales beneficiados } & \multicolumn{3}{|l|}{ Hacer trabajo voluntario } \\
\hline de recuperarlo & 31 & 11.5 & & 192 & 71.7 \\
\hline \multicolumn{3}{|l|}{ Es su responsabilidad por } & \multirow{2}{*}{\multicolumn{3}{|c|}{$\begin{array}{l}\text { No es necesario que dé o haga } \\
\text { algo }\end{array}$}} \\
\hline \multirow{2}{*}{$\begin{array}{l}\text { haberlo contaminado } \\
\text { Tendrían más compromiso }\end{array}$} & 23 & 8.5 & & 56 & 20.7 \\
\hline & 7 & 2.6 & \multirow{2}{*}{$\begin{array}{l}\text { Pagar un impuesto especial } \\
\text { Otra }\end{array}$} & 16 & 5.6 \\
\hline Conocen el río & 6 & 2.2 & & 6 & 2 \\
\hline \multirow{2}{*}{$\begin{array}{l}\text { Se requiere su cooperación } \\
\text { Otras }\end{array}$} & 6 & 2.2 & \multirow[t]{2}{*}{ Total } & \multirow[t]{2}{*}{270} & \multirow{2}{*}{100} \\
\hline & 13 & 4.8 & & & \\
\hline No aplica & 184 & 68.1 & \multicolumn{3}{|c|}{ ¿Por qué cree que el río estará limpio? (O Bis) } \\
\hline \multirow[t]{3}{*}{ Total } & \multirow{3}{*}{270} & \multirow{3}{*}{100} & \multirow[t]{2}{*}{ Respuesta } & \multirow{2}{*}{$\begin{array}{l}\text { Frecuencia } \\
\text { absoluta }\end{array}$} & \multirow{2}{*}{$\begin{array}{l}\text { Porcentaje } \\
\quad(\%)\end{array}$} \\
\hline & & & & & \\
\hline & & & Tengo la esperanza & 82 & 30.4 \\
\hline \multicolumn{3}{|c|}{$\begin{array}{l}\text { O. ¿Cree que realmente el río Magdalena estará limpio } \\
\text { algún día? }\end{array}$} & $\begin{array}{l}\text { Es un patrimonio histórico y } \\
\text { natural de la ciudad }\end{array}$ & 34 & 12.6 \\
\hline \multirow[t]{2}{*}{ Respuesta } & Frecuencia & Porcentaje & \multirow[t]{2}{*}{ Nos beneficiará a todos } & & \\
\hline & absoluta & $(\%)$ & & 33 & 12.2 \\
\hline \multirow[t]{2}{*}{ Sí } & & & \multirow{2}{*}{$\begin{array}{l}\text { Tiene una gran potencial para ser } \\
\text { un espacio público }\end{array}$} & & \\
\hline & 188 & 69.6 & & 19 & 7.0 \\
\hline No & 82 & 30.4 & Otras & 20 & 7.4 \\
\hline \multirow[t]{2}{*}{ Total } & 270 & 100 & No aplica & 82 & 30.4 \\
\hline & & & Total & 270 & 100 \\
\hline
\end{tabular}

Fuente: Elaboración propia con base en el trabajo de campo. N/C = no contestó

M. ¿Quién es el actor más confiable para recuperar el río?

\begin{tabular}{lcc} 
Respuesta & $\begin{array}{c}\text { Frecuencia } \\
\text { absoluta }\end{array}$ & $\begin{array}{c}\text { Porcentaje } \\
(\%)\end{array}$ \\
\cline { 2 - 3 } Vecinos & 86 & 31.9 \\
Todos & 65 & 24.1 \\
& 53 & 19.6 \\
Científicos & 29 & 10.7 \\
Comunidades rurales & 12 & 4.4 \\
Políticos & 24 & 8.9 \\
Otros & 1 & 0.4 \\
N/C & 270 & 100 \\
Total & & \\
\hline
\end{tabular}

N. ¿Qué estaría dispuesto a dar o hacer si eso garantiza la limpieza del río?

Hacer trabajo voluntario

No es necesario que dé o haga

Pagar un impuesto especial

Otra

¿Por qué cree que el río estará limpio? (O Bis) responsables por su falta de capacidad y eficacia para solucionar los problemas ambientales de las delegaciones. De hecho, los identifican como responsables directos del crecimiento desordenado de la ciudad, ya sea por prácticas clientelares que favorecen a los asentamientos irregulares o por la falta de planeación que no incorpora el tema ambiental entre sus prioridades. Finalmente, la percepción sobre los asentamientos irregulares es ambivalente. Por un lado, se reconoció el impacto negativo de ocupar suelo de conservación debido a la tala de árboles, la acumulación de basura en la parte alta de la microcuenca y la contaminación tanto del agua como del suelo por aguas servidas. Por el otro, se considera que los asentamientos irregulares son resultado de problemas estructurales como la desigualdad socioeconómica que impide adquirir una vivienda a precios accesibles al interior de la ciudad. 
CUADRO IV. PRUEBAS DE HIPÓTESIS CHI-CUADRADO

\begin{tabular}{lrrrrr}
\hline \multirow{2}{*}{ Pregunta } & \multicolumn{5}{c}{ Variable } \\
\cline { 2 - 6 } & $\begin{array}{c}\text { Ubicación en } \\
\text { cuenca }\end{array}$ & Edad & Sexo & Escolaridad & $\begin{array}{r}\text { Tiempo de } \\
\text { residencia }\end{array}$ \\
\hline $\begin{array}{l}\text { B. Afectación por } \\
\text { contaminación del río }\end{array}$ & $6.574(.010)$ & $.707(.400)$ & $2.194(.139)$ & $3.750(0.53)$ & $.001(.979)$ \\
$\begin{array}{l}\text { D. Corresponsabilidad en la } \\
\text { contaminación del río }\end{array}$ & $2.945(.086)$ & $6.139(.013)$ & $9.620(.002)$ & $1.388(.239)$ & $4.067(.044)$ \\
$\begin{array}{l}\text { E. Sabe del plan } \\
\text { K. Ha hecho algo por el río }\end{array}$ & $24.314(.000)$ & $1.214(.271)$ & $1.066(.302)$ & $.654(.419)$ & $.034(.854)$ \\
O. Río limpio en futuro & $37.698(.000)$ & $13.699(.000)$ & $.283(.595)$ & $1.201(.273)$ & $2.920(.088)$ \\
\hline
\end{tabular}

Fuente: Elaboración propia con base en trabajo de campo. Nivel de significancia: 0.05 (valor p), Grados de libertad: 1, Valor crítico: 3.8415. Entre paréntesis se identifica el estadístico de probabilidad. Estadísticos de prueba obtenidos mediante la continuidad de Yates

Poco menos de la mitad de los encuestados asumieron ser parcialmente corresponsables en la contaminación del río (46.7\%), quienes asumieron dicha responsabilidad, señalaron que eventualmente habían tirado basura en el cauce o sus márgenes $(24.8 \%)$, no habían hecho nada para presionar a las autoridades para limpiar el río o incluso no habían amonestado verbalmente cuando vieron que alguien lo contaminaba ( $15.2 \%$ ) y porque estaban conscientes de la alta probabilidad de que las aguas residuales de su vivienda descargan en el río (5.9\%). Resulta interesante que los encuestados perciben que su silencio ante la contaminación también daña el río por no asumir una defensa activa. La corresponsabilidad está asociada estadísticamente con las variables de la edad, el sexo y tiempo residencia, de manera que las mujeres jóvenes y que tienen menos de 10 años de residencia en la microcuenca son más propensas a asumir una corresponsabilidad por la contaminación del río. Por su parte, el sector que percibió no tener responsabilidad alguna en la contaminación del río, afirmó que cuidan el ambiente (v. gr. ponen la basura en su lugar, $40.7 \%$ ) o bien, porque el gobierno y los habitantes de las márgenes son quienes deben asumir la responsabilidad de cuidarlo (9.6\%).

La encuesta señala que $70.7 \%$ desconocía la existencia e implementación del Plan Maestro. En este rubro hay una fuerte asociación entre el conocimiento del Plan y la ubicación del encuestado en la microcuenca. Resulta comprensible que en la parte baja fuera prácticamente nulo el conocimiento sobre el Plan, ya que hasta la fecha no se ha realizado ninguna acción en esa zona. Las personas que respondieron estar enterados del Plan en la parte alta argumentaron saberlo porque algún familiar, vecino o amigo le informó sobre el proyecto (15.6\%), no recordaban exactamente el medio por el que se enteraron (6.3\%) o habían observado directamente los avances de las obras $(5.6 \%)$.

En la dimensión valorativa, los encuestados señalaron que la acción más importante que debe implementarse para recuperar el río Magdalena es el mejoramiento de la educación ambiental (39.6 \%), fomentar los mecanismos de participación ciudadana (14.1\%) y desarrollar las dos acciones simultáneamente $(30.7 \%)$. Las menciones sobre la importancia de la educación ambiental fueron más frecuentes en la parte alta y por parte de los jóvenes. Se mencionó que esta medida era indispensable porque desde su punto de vista la mayoría de las acciones que realizan los vecinos son contrarias a la sustentabilidad del río, hecho que relacionan con la falta de elementos cognitivos y valorativos que le impiden al sujeto reconocer el daño que provoca tirar basura en el bosque y en el río, causar lesiones al arbolado o construir su vivienda en la planicie de inundación. De hecho, se indicó que la educación ambiental tendría una repercusión positiva en la participación ciudadana, ya que en la medida que el ciudadano tenga una mayor consciencia y sensibilidad sobre los problemas ambientales, aumentará la cantidad de vecinos dispuestos a participar, además tendrán mayores herramientas para proponer soluciones. En otras medidas $(2.6 \%)$ se mencionó evitar la descarga de aguas residuales, recuperar la planicie de inundación y que los habitantes de la microcuenca asuman un compromiso real para no ensuciar el río. Los encuestados indicaron que para participar en la recuperación del río es necesario recibir una invitación del gobierno (62.5\%) o tener más tiempo libre 
(18.5\%). Los encuestados que señalaron innecesaria su participación $(11.5 \%)$ argumentaron que la tarea de limpiar el río era responsabilidad exclusiva del gobierno y de los vecinos que están más próximos al cauce a quienes les afecta directamente la contaminación. En otras respuestas $(7.8 \%)$ se mencionó la posibilidad de involucrarse en las tareas de limpieza en la medida en que fuera una política obligatoria, si la autoridad expusiera públicamente un plan antes de solicitar la participación de los ciudadanos, o bien, que sólo se sumaría si observa previamente avances reales en la recuperación del río.

La principal ventaja de participar en los espacios abiertos por la autoridad para la participación ciudadana consiste en la posibilidad de proponer directamente acciones para mejorar las condiciones del río (38.1\%). Esta respuesta fue más frecuente en la parte baja, en los encuestados mayores de 50 años, quienes cuentan con estudios equivalentes al bachillerato o una carrera técnica y tienen más de 30 años de residir en la microcuenca. En segundo lugar, se valoró que los ciudadanos puedan expresar su voz en torno al problema ambiental $(21.5 \%)$ y en tercero por la posibilidad de avalar los proyectos que la autoridad quiere implementar (18.1 $\%$ ). Los encuestados aseveraron que los espacios de participación ciudadana constituyen oportunidades de aprendizaje ambiental para que los concurrentes adquieran conocimientos sobre el estado del río y del bosque, los cuales serían de utilidad para modificar prácticas que resultan contrarias a la sustentabilidad. Por el contrario, entre las desventajas de participar se encuentran la incertidumbre de asistir a espacios manipulados por la autoridad en los que las propuestas ciudadanas no sean recuperadas como el eje central de la política de rescate $(25.6 \%$ de las menciones). Los jóvenes, las mujeres y los encuestados con estudios equivalentes al bachillerato fueron quienes más señalaron esta desventaja de la participación. Las siguientes desventajas que se indicaron con mayor frecuencia fueron pelearse con los vecinos $(22.6 \%)$, la pérdida de tiempo libre (18.5\%) y el pesimismo de que inevitablemente el río volvería a contaminarse en el futuro $(4.4 \%)$.

En la dimensión de actitudes resalta que la mayor parte de la población afirmó no haber realizado previamente algún tipo de actividad dirigida a mejorar la calidad del río (53\%). Los ciudadanos que respondieron haber hecho alguna acción señalaron principalmente no tirar basura en el cauce o en las cercanías, haber amonestado verbalmente a familiares o vecinos que la estaban tirando, barrer sus banquetas, separar PET y levantar basura de la acera para que ésta no caiga en el río. En este rubro se identificó una asociación estadística entre ubicación en la cuenca y edad con realización de actividades a favor de la limpieza del río, de manera que los adultos que habitan en la parte baja serían más propensos a realizar alguna actividad que desde su perspectiva favorece al río, aunque en el sentido acotado de manejar responsablemente los residuos sólidos en la vía pública. Prácticamente ocho de cada 10 encuestados señaló que la clase política era el actor menos confiable para recuperar el río. Entre los principales argumentos que esgrimieron para justificar su respuesta destaca la ínfima eficacia y competencia laboral de los gobernantes para cumplir sus compromisos $(28.9 \%)$. En segundo lugar, se mencionó que los políticos privilegian su interés privado sobre las necesidades y la defensa del bien público (22.6\%). Varios encuestados señalaron que para ellos la clase política utiliza sus puestos de representación popular para buscar otro escaño que permita alargar su carrera política y que esto los termina alejando de las necesidades de los representados. Finalmente, se destaca un proceso de "naturalización" de la desconfianza frente a este actor, ya que cuando se les pidió justificar su elección, varios encuestados respondieron que "nadie confía en los políticos" o "porque tienen mala reputación" $(13.7 \%)$. Por el contrario, los vecinos resultaron la opción más seleccionada como los actores más confiables para dirigir las tareas de recuperación $(31.9 \%)$, particularmente por las mujeres y los encuestados con estudios de licenciatura. La selección de esta respuesta se argumentó porque los vecinos serían los principales interesados en que realmente se limpie el río, ya que en este momento son los principales afectados por las externalidades negativas de la contaminación ( $11.5 \%$ de quienes señalaron a este actor). Otro argumento consistió en que el actor que contamina tiene un deber ético de solucionar el problema que ha generado, es decir, si los vecinos son los que contaminan, entonces son ellos quienes deberían reparar el daño $(8.5 \%)$. Sobre la cuestión de qué estaría dispuesto a dar el ciudadano si dicha contribución fuera definitiva para asegurar la limpieza del río, $71.1 \%$ respondió hacer trabajo voluntario, mientras que $20.7 \%$ respondió que no era necesario dar o hacer algo. Finalmente, los encuestados fueron optimistas con respecto al futuro del río, ya que $69.6 \%$ declaró que confía en que el río Magdalena estará limpio algún día, esta confianza radica en una simple esperanza del tipo "así lo espero o lo deseo" $(30.4 \%)$, porque finalmente se reconocerá como un patrimonio histórico y natural de la ciudad (12.6\%) o porque los habitantes de la ciudad se convencerán de que su limpieza constituye un beneficio colectivo 
para todos $(12.2 \%)$. El optimismo está relacionado estadísticamente con la ubicación en la cuenca y la edad, de manera que los adultos que habitan la parte baja de la microcuenca consideran que el río estará limpio en un futuro.

\section{DISCUSIÓN}

A diferencia de otras investigaciones estadísticas en las cuales se ha encontrado fuertes correlaciones entre variables demográficas y las preocupaciones por el ambiente (Jones y Dunlap 1992, Scott y Willits 1994), la presente encuesta no muestra constantes significativas con respecto a la perspectiva ambiental que hay sobre el río, ya que éstas varían de acuerdo con la dimensión analítica que se analiza. Así, en contraste con encuestas que señalan a los jóvenes como el sector que se preocupa más por el ambiente (Fransson y Gärling 1999), en nuestro caso hay reactivos en los cuales los adultos mayores a 45 años tienen una mayor frecuencia, como es el caso de quienes declararon haber hecho algo por contribuir en la limpieza del río o quienes tienen mayor confianza en que se podrá recuperar en el futuro. No obstante, los jóvenes son efectivamente quienes asumen una mayor corresponsabilidad en el deterioro del río, están más convencidos de que un cambio significativo radica en mejorar la educación ambiental de los habitantes de la microcuenca y en que los espacios de participación deben recuperar la voz ciudadana como un elemento central de cualquier proyecto dirigido a su recuperación.

A nivel general, sobresale el hecho que la mayoría siente algún tipo de afectación por la contaminación del río Magdalena, pero menos de la mitad asumió tener algún tipo de responsabilidad en la generación de los problemas ambientales ni haber realizado alguna actividad previa dirigida a mejorarlo. Este resultado resulta muy consistente con la Encuesta Nacional sobre Medio Ambiente (ENMA) en el cual se identifica que la ciudadanía declara estar interesada en los problemas ambientales y tener una buena disposición para coadyuvar en la solución. Sin embargo, esta disposición contrasta con las actividades concretas que se hacen con relación al ambiente, ya que sólo dos de cada de 10 realiza la mayoría de sus acciones teniendo en cuenta a la naturaleza (IIJ-UNAM 2012). Este hecho revela que el tema ambiental importa como un asunto de interés público, pero son pocos quienes efectivamente se involucran en proyectos específicos como puede ser la recuperación de un río urbano. Esta disonancia entre la dimensión cognitiva y la práctica social se puede explicar a partir de otros resultados que se obtuvieron en la encuesta. En primer lugar destaca que, a pesar de la alta valoración ciudadana que se deposita en la participación como un mecanismo para resolver la contaminación del río, se desconfía notoriamente de los espacios participativos que abren las autoridades de gobierno por considerarlos prácticas de manipulación en donde se legitiman decisiones previamente tomadas por las propias autoridades, esto es, no están realmente abiertos a incorporar la voz ciudadana como eje articulador de los proyectos. La desconfianza en la clase política (representantes y partidos) es una tendencia a la alza en los últimos años. Diferentes encuestas nacionales suelen colocar a dichas instituciones como las últimas en el rubro de confianza ciudadana. Por ejemplo, en la última Encuesta Nacional de Cultura Política y Prácticas Ciudadanas, ENCUP (Segob 2012) las organizaciones políticas con más baja calificación en una escala sobre diez fueron la policía (4.7), los partidos políticos (4.8) y los senadores (4.9), mientras que en la ENMA los últimos lugares fueron ocupados por la policía (4.9), los partidos políticos (5.1) y los diputados federales (5.2). La desconfianza institucional coincide con la baja capacidad que asume la ciudadanía a nivel nacional para influir efectivamente en las acciones del gobierno. En la Encuesta Nacional sobre Calidad de la Ciudadanía (IFE 2014) se reveló que $70.9 \%$ de la ciudadanía declaró no tener la posibilidad de influir realmente en las acciones de gobierno. En suma, se desconfía de los espacios de participación que abre la autoridad por su bajo impacto en la toma de decisiones, aspecto que retroalimenta la percepción ciudadana referente a su escasa capacidad de influir en las acciones gubernamentales y que tendencialmente los aleja más de acudir a dichos espacios. Estos resultados son preocupantes si se considera que la confianza institucional es indispensable para que el gobierno pueda activar y mantener espacios de participación ciudadana dirigidos a la solución de problemas ambientales. En la recuperación de ríos urbanos es indispensable el papel activo del gobierno en su diseño e implementación, pero será muy complicado que pueda encontrar el apoyo y la colaboración ciudadana si preexiste una relación tan marcada de desconfianza hacia ellos. Ahora bien, cuando la ciudadanía responde que lo que necesita para involucrarse en la recuperación del río es fundamentalmente una invitación del gobierno, asume que este tipo de proyectos surgen de la esfera gubernamental, como un acto de autoridad que moviliza recursos económicos y humanos para la salvaguarda 
de un bien público. También implica la necesidad de reconocimiento que tiene la ciudadanía para que el gobierno la valore como un interlocutor indispensable en la toma de decisiones sobre el ambiente.

En segundo lugar resulta contrastante que la recuperación del río se deposita principalmente en manos de la ciudadanía o comunidades locales, pero al mismo tiempo se reconoce como principal desventaja de la participación ciudadana la dificultad de lograr consensos, o como lo dijeron los propios encuestados, participar significa para ellos "pelearse con los vecinos". Esta respuesta coincide con que $44 \%$ de la población nacional considera difícil organizarse con otros ciudadanos para trabajar juntos en aras de resolver un problema común (Segob 2012). Si se revisan los argumentos detrás de la "confianza" que los encuestados depositan en los vecinos para dirigir las tareas de rescate, los de mayor frecuencia son de carácter pragmático, ya que los encuestados señalaron que son los vecinos quienes padecen directamente las externalidades de la contaminación, por lo que serían los principales interesados en revertirla y en transformar el río en un espacio público limpio que provea servicios ecosistémicos para la zona. Aunque se trate de un bien ambiental ampliamente valorado en el territorio, la ciudadanía percibe como una tarea difícil organizarse colectivamente para recuperar el río. Esta valoración coincide con análisis técnicos que mencionan la importancia de la escala en la generación de consensos en torno a un bien común, ya que entre mayor sea el espacio territorial de planeación (en este caso una cuenca), aumentará la pluralidad y heterogeneidad de perspectivas ambientales e intereses sobre la manera de gestionar el río, así como otros recursos naturales y urbanos asociados al cauce (Jones 1999, Zamora y González 2014). Por ello se ha insistido en que la participación ciudadana puede lograr sus objetivos de generar acción colectiva en la medida en que estos sean esfuerzos que se mantengan en el tiempo hasta que se alcancen los consensos mínimos en torno a la recuperación del río (Otto et al. 2004). Sólo con un diálogo sostenido es posible que los diferentes actores tengan una visión más integral sobre la complejidad de los problemas socioambientales que caracterizan a las cuencas, de manera que efectivamente los mecanismos de participación también cumplan su papel de espacios para la educación ambiental (tal y como lo consideraron los encuestados) y para la propia vida democrática (Font et al. 2012). En ese sentido, la ciudadanía considera que los espacios de participación pueden ser oportunidades para el aprendizaje, no sólo referente a las características del río, sus problemas ambientales y posibles maneras de recuperarlo, sino también para aprender a tomar decisiones colectivamente.

\section{CONCLUSIONES}

A pesar de la inercia histórica que ha tenido la ciudad en tratar a los ríos urbanos como drenajes para reconvertirlos en vialidades, el río Magdalena presenta una gran oportunidad para transformar este paradigma sanitarista gracias a la importancia que tiene en la provisión de agua potable y otros servicios ecosistémicos para el surponiente de la ciudad, sin olvidar los espacios públicos a los que está vinculado en el suelo urbano como el Foro Cultural Contrerense y los Viveros de Coyoacán. La encuesta reveló que los habitantes de la microcuenca no son indiferentes al presente y futuro del río, es un tema que les preocupa dado que lo perciben como un patrimonio ambiental de la ciudad constantemente amenazado por la expansión urbana descontrolada. A pesar de reconocer la contaminación del río como un problema ambiental que requiere solución, resulta una minoría el sector de la población que se asume como parte del problema o que afirma realizar acciones para contribuir en su recuperación. Esta tendencia coincide con encuestas a nivel nacional en las que se identifica como la alta valoración de los problemas ambientales no se ve reflejada en las prácticas sociales cotidianas o de carácter organizativo para intentar solucionarlos. En la microcuenca del río Magdalena dicha situación se complementa con las perspectivas que se tienen sobre el entorno social y político, ya que la participación ciudadana en la recuperación del río está atravesada por una baja confianza en el gobierno, particularmente en los espacios deliberativos y participativos que abren en el marco de sus políticas ambientales y urbanas. El hallazgo no es menor, ya que la recuperaciones más exitosas de ríos urbanos han tenido canales de participación plurales y continuos que permiten recuperar las preferencias ciudadanas como premisa básica de la factibilidad social y política de tales iniciativas. Una manera de restaurar los lazos sociales entre sociedad civil y gobierno en la recuperación de un río y en cualquier política ambiental que involucre las demandas ciudadanas, consiste en la transparencia con la que se maneja la información relacionada al proyecto, lo que implica compartir el conocimiento que se genera sobre la microcuenca entre todos los interesados (Gardiner et al. 1994), incluidos los aspectos financieros y los propios mecanismos de participación que se implementarán (¿Cuáles son sus objetivos? ¿Durante cuánto tiempo funcionarán? ¿A quiénes se invita a participar? 
¿Por qué? etc.). De lo contrario, persistirá el hecho de contar con ciudadanos interesados en resolver la contaminación del río, pero profundamente escépticos de poder influir y ver reflejada su voz en estas iniciativas.

Esta investigación constituye un primer ejercicio para conocer las perspectivas ambientales que tienen los habitantes de una microcuenca sobre la contaminación y la recuperación de un río urbano. Es importante estudiar otros casos en el país para identificar con mayor claridad las particularidades del río Magdalena y detectar tendencias más generales que permitan conocer las percepciones, los valores y las actitudes sobre el entorno natural y sociopolítico de los ríos urbanos, lo cual constituye un elemento sine qua non para involucrar efectivamente a la ciudadanía en su recuperación y por ende en la construcción de ciudades sostenibles.

\section{AGRADECIMIENTOS}

Los autores le agradecen a Verónica Aguilar Zamora del Laboratorio de Ecosistemas de Montaña de la Facultad de Ciencias de la UNAM por su apoyo en la elaboración del mapa. También agradecemos a Irma Flores y Joaquín Hernández por su invaluable trabajo en el levantamiento de la encuesta, así como a Alba Campos en la sistematización de la misma. El Dr. Zamora agradece al Programa de becas posdoctorales de la Universidad Nacional Autónoma de México (UNAM) por el apoyo para la elaboración del presente artículo.

\section{REFERENCIAS}

Almeida-Leñero L., Nava M., Ramos A., Espinosa M., Ordoñez M. y Jujnovsky J. (2007). Servicios ecosistémicos en la cuenca del río Magdalena, Distrito Federal, México. Gaceta Ecológica 84-85 (edición especial), 53-64.

Asakawa S., Yoshida K. y Yabe K. (2004). Perceptions of urban stream corridors within the greenway system of Sapporo, Japan. Landscape Urban Plan. 68 (2-3), 167182. DOI: $10.1016 / \mathrm{S} 0169-2046(03) 00158-0$

Benez M.C., Kauffer E.F. y Álvarez G.C. (2010). Percepciones ambientales de la calidad del agua superficial en la microcuenca del río Fogótico, Chiapas. Frontera Norte 22 (43), 129-158.

Brody S.D., Highfield W. y Alston L. (2004). Does location matter? Measuring environmental perceptions of creeks in two San Antonio watersheds. Environ. Behav. 36 (2), 229-250. DOI: 10.1177/0013916503256900
Burningham K. y Cooper G. (1999). Being constructive: Social constructionism and the environment. Sociology 33 (2), 297-316. DOI: 10.1177/S0038038599000280

Carmona J., Ramírez R., Bojorge M., González B. y Cantoral E. (2016). Estudio del valor indicador de las comunidades de algas bentónicas: una propuesta de evaluación y aplicación en el río Magdalena, Ciudad de México. Rev. Int. Contam. Ambie. 32 (2), 139-152. DOI: 10.20937/RICA.2016.32.02.01

Dufour S. y Piégay H. (2009). From the myth of a lost paradise to targeted river restoration: forget natural references and focus on human benefits. River Research and Applications 25 (5), 568-581.

DOI: $10.1002 /$ rra.1239

Durand L. (2008). De las percepciones a las perspectivas ambientales. Una reflexión teórica sobre la antropología y la temática ambiental. Nueva Antropología 21 (68), 75-87.

Eagly A.H. y Chaiken S. (1993). The psychology of attitudes. Harcourt Brace Jonanovich College Publishers, Orlando, EUA. 794 pp.

Feld C.K., Birk S., Bradley D.C., Hering D., Kail J., Marzin A., Melcher A., Nemitz D., Pedersen M.L., Pletterbauer F., Pont M., Verdonschot P.F. y Friberg N. (2011). Chapter three - From natural to degraded rivers and back again: a test of restoration ecology and practices. Adv. Ecol. Res. 44, 119-209.

DOI: 10.1016/b978-0-12-374794-5.00003-1

Figuereido E., Valente S., Coelho C. y Pinho L. (2009). Coping with risk: analysis on the importance o integrating social perceptions on flood risk into management mechanisms - the case of the municipality of Águeda, Portugal. J. Risk Res. 12 (5), 581-602. DOI: 10.1080/13669870802511155

Font J., Blanco I., Gomà R. y Jarque M. (2012). Mecanismos de participación ciudadana en la toma de decisiones locales: Una visión panorámica. Transformación, Estado y Democracia 7 (50), 102-31.

Fransson N. y Gärling T. (1999) Environmental concern: Conceptual definitions, measurement methods, and research findings. J. Environ. Psychol. 19 (4), 369-382.

Freudenburg W.R., Frickel S. y Gramling, R. (1995). Beyond the nature/society divide: Learning to think about a mountain. Sociol. Forum 10 (3), 361-392.

Gardiner J., Thomson K. y Newson M. (1994). Integrated watershed/river catchment planning and management: a comparison of selected Canadian and United Kingdom experiences. J. Environ. Plann. Man. 37 (1), 53-67. DOI: 10.1080/09640569408711959

Gobster P.H. (1998). Nearby neighborhood residents' images and perceptions of the river. En: People and the river: Perception and use of Chicago waterways 
for recreation (P.H. Gobster y L.M. Westphal, Ed.). National Park Service, Rivers, Trails, and Conservation Assistance Program, Milwaukee, Wisconsin, EUA, pp. 5-48.

Gould K. (1993). Pollution and perception: Social visibility and local environmental mobilization. Qual. Sociol. 16 (2), 157-178.

DOI: $10.1007 / \mathrm{BF} 00989748$

Green C.H. y Tunstall S.M. (1992). The amenity and environmental value of river corridors in Britain. En: River conservation and management. (P.J. Boon, P. Carlow y G.E. Petts, Ed.) Wiley, Chichester, Inglaterra, pp. 425-441.

Guagnano G.A. (1995). Locus of control, altruism and agentic disposition. Popul. Environ. 17 (1), 63-77. DOI: $10.1007 / \mathrm{BF} 02208278$

Hannigan J. (2006). Environmental sociology. 2a ed., Routledge, Nueva York, EUA. 194 pp.

House M. y Fordham M. (1997). Public perceptions of river corridors and attitudes toward river works. Landscape Res. 22 (1), 25-44.

DOI: $10.1080 / 01426399708706499$

IFE (2014). País sobre la calidad de la ciudadanía en México. Informe. Instituto Federal Electoral. Documento analítico de la Encuesta. Distrito Federal, México, $277 \mathrm{pp}$.

IIJ-UNAM (2012). Encuesta nacional de percepciones y actitudes hacia el medio ambiente (ENMA). Instituto de Investigaciones Jurídicas - Universidad Nacional Autónoma de México. Encuesta (principales resultados). Distrito Federal, México, 73 pp.

Ímaz M.A., González M., Gutiérrez J.L., Vargas P., Camacho R., Ayala D., Anglés M., Ibarra R. y Beristain A.G. Presentación. En: La dimensión ambiental en los albores del siglo XXI. Miradas desde la diversidad (M.A. Ímaz, Coord.), Universidad Nacional Autónoma de México-Instituto de Investigaciones Jurídicas, Distrito Federal, México, pp. 29-32.

Jones R.E. y Dunlap R.E. (1992). The social bases of environmental concern: Have they changed over time? Rural Sociol. 57 (1), 28-47.

DOI: 10.1111/j.1549-0831.1992.tb00455.x

Jones S. (1999) Participation and community at the landscape scale. Landscape Journal 18 (1), 65-78.

DOI: $10.3368 / \mathrm{lj} .18 .1 .65$

Legorreta J. (2009). Ríos, lagos y manantiales del Valle de México. Universidad Autónoma Metropolitana, Gobierno del Distrito Federal, Ciudad de México, México, 365 pp.

Lundy L. y Wade R. (2011). Integrating sciences to sustain urban ecosystem services. Prog. Phys. Geog. 35 (5), 653-669.

DOI: $10.1177 / 0309133311422464$
Macnaghten P. y Urry J. (1995). Towards a sociology of nature. Sociology 29 (2), 203-220.

DOI: $10.1177 / 0038038595029002002$

Mohai P. (1985). Public concern and elite involvement in environmental-conservation issues. Soc. Sci. Quart. 66 (4), 820-838.

Norton B. y Hannon B. (1997). Environmental values: a place-based approach. Environ. Ethics 19 (3), 227-245. DOI: 10.5840 /enviroethics 199719313

Otto B., McCormick K. y Leccese M. (2004). Ecological riverfront design: Restoring rivers, connecting communities. American Planning Association, Chicago, EUA, 177 pp.

Pahl-Wostl C. (2006). The importance of social learning in restoring the multifunctionality of rivers and floodplains. Ecol. Soc. 11 (1), 10.

Petty R.E., Wegener D.W. y Fabrigar L.R. (1997). Attitudes and attitude change. Annu. Rev. Psychol., 48, 609-647. DOI: 10.1146/annurev.psych.48.1.609

Pérez C. (2005). Muestreo estadístico: Conceptos y problemas resueltos. Pearson, Madrid, España, 392 pp.

Perló M. (2010). La apuesta para el desarrollo inmobiliario: recuperación de ríos urbanos. En: Rescate de ríos urbanos. Propuestas conceptuales y metodológicas para la restauración y rehabilitación de ríos. (A.E. González, L. Hernández, M. Perló e I. Zamora, Eds.). PUEC-UNAM, Distrito Federal, México, pp. 50-54

Perló M. y González A.E. (2006). Del agua amenazante al agua amenazada. Cambios en las representaciones sociales de los problemas del agua en el Valle de México. En: Más allá del cambio climático. Las dimensiones psicosociales del cambio ambiental global . (J. Urbina y J. Martínez, Coords.). Secretaría de Medio Ambiente y Recursos Naturales, Instituto Nacional de Ecología, Facultad de Psicología-UNAM, Distrito Federal, México, pp. 47-64.

Perló M. y González A.E. (2009). El papel de la interdisciplinariedad científica y de la participación social en el rescate de ríos urbanos. En: Gestión del agua: una visión comparativa entre México y Brasil (I. Sandré, R. Luiz do Carmo, S. Vargas y N.B. Guzmán, Eds.). Archivo histórico del Agua, Instituto Mexicano de Tecnología del Agua, Universidad Autónoma del Estado de Morelos, Jiutepec, Morelos, México, pp. 177-188.

Petts J. (2006). Managing public engagement to optimize learning: Reflections from urban river restoration. Hum. Ecol. Rev. 13 (2), 172-181.

Petts J. (2007). Learning about learning: Lessons from public engagement and deliberation on urban river restoration. Geogr. J. 173 (4), 300-311.

DOI: $10.1111 / j .1475-4959.2007 .00254 . x$ 
Postel S. y Richter B. (2003). Rivers for life: Managing water for people and nature. Island Press, Washington, EUA, 220 pp.

Riley A. L. (1998). Restoring streams in cities: A guide for planners, policymakers, and citizens. Island Press, Washington, EUA, 448 pp.

Ryan R. (1998). Local perceptions and values for a midwestern river corridor. Landscape Urban Plan. 42 (2-4), 225-237.

DOI: $10.1016 / \mathrm{S} 0169-2046(98) 00089-9$

Scott D. y Willits F. (1994). Environmental attitudes and behavior. A pennsylvania survey. Environ. Behav. 26 (2), 239-260.

DOI: $10.1177 / 001391659402600206$

Sedema y UNAM (2009). Plan de Manejo Integral y Aprovechamiento Sustentable de la Cuenca del río Magdalena. Plan Maestro. Secretaría del Medio Ambiente del Gobierno del Distrito Federal - Universidad Nacional Autónoma de México. Distrito Federal, México, $267 \mathrm{pp}$.

Segob (2012). Encuesta nacional sobre cultura política y prácticas ciudadanas (ENCUP). Secretaría de Gobernación. Encuesta. Distrito Federal, México [en línea] http://www.encup.gob.mx/en/Encup/Quinta_ENCUP_2012 15/10/2015

Sutton P.W. (2004). Nature, environment and society. Palgrave Macmillan, Nueva York, EUA, 216 pp.

Tapsell S. (1995). River restoration: what are we restoring to? A case study of the Ravensbourne River, London. Landscape Res. 20 (3), 98-111. DOI: $10.1080 / 01426399508706464$
Tunstall S.M., Penning-Rowsell E.C., Tapsell S.M. y Eden S.E. (2000). River restoration: Public attitudes and expectations. Water Environ. J. 14 (5), 363-370. DOI: 10.1111/j.1747-6593.2000.tb00274.x

Tunstall S.M., Tapsell S.M. y Fordham M. (1994). Public perception of rivers and flood defence: Final Report. Resumen ejecutivo. National Rivers Authority y Flood Hazard Research Centre, Middlesex University. Middlesex, Inglaterra, $15 \mathrm{pp}$.

Valencia A., Arias M. y Vázquez R.(2010). Opiniones y actitudes. Ciudadanía y conciencia medioambiental en España. Centro de Investigaciones Sociológicas, Madrid, España, 102 pp.

Wohl E., Angermeier P., Bledsoe B., Kondolf M., MacDonnell L., Merritt D., Palmer M., Poff N. y Tarboton D. (2005). River restoration. Water Resour. Res. 41 (10), 1-12. DOI: 10.1029/2005WR003985

Zamora I. y González A.E. (2014). Representaciones sociales del territorio en el rescate de ríos urbanos: La experiencia de la integración del Plan Maestro de Rescate del Río Magdalena, Ciudad de México. En: Estado y ciudadanías del agua. Cómo significar las nuevas relaciones (F.de Alba y L. Amaya, Coords.). Universidad Autónoma Metropolitana, Unidad Cuajimalpa, Distrito Federal, México, pp.105-151. 